\title{
Numerical Statistic Approach for Peak Factor of Non-Gaussian Wind Pressure on Building Claddings
}

\author{
Tao Ye ${ }^{1},,^{1,2,3}$ Ledong Zhu, ${ }^{1,2,3}$ and Zhongxu Tan ${ }^{1}$ \\ ${ }^{1}$ State Key Laboratory of Disaster Reduction in Civil Engineering, Tongii University, Shanghai 200092, China \\ ${ }^{2}$ Key Laboratory of Transport Industry of Bridge Wind Resistance Technology (Shanghai), Tongji University, \\ Shanghai 200092, China \\ ${ }^{3}$ Department of Bridge Engineering, Tongji University, Shanghai 200092, China
}

Correspondence should be addressed to Tao Ye; yetao1982827@163.com

Received 11 December 2019; Revised 21 March 2021; Accepted 15 April 2021; Published 5 May 2021

Academic Editor: Pier Paolo Rossi

Copyright (c) 2021 Tao Ye et al. This is an open access article distributed under the Creative Commons Attribution License, which permits unrestricted use, distribution, and reproduction in any medium, provided the original work is properly cited.

\begin{abstract}
Wind-induced pressures on high-rise buildings claddings are mostly non-Gaussian distribution, and there is a one-to-one relationship between a specified guarantee rate and its corresponding peak factor. In this study, a stepwise search method for calculating the peak factor of non-Gaussian wind pressure and a gradual independent segmentation method for extracting independent peak values are proposed to determine the relationship accurately. Based on the pressure data of a high-rise building obtained from a rigid model wind tunnel test, the peak factors of non-Gaussian wind pressures on claddings are calculated and compared by using several typical methods. The value of the peak factor and its error rate calculated by several methods is compared with the observed average peak value, and the conversion between the guaranteed rate and the peak factor is discussed. Based on the reliability theory, the true distribution of wind pressure time history was approached infinitely through an efficient numerical method in the process of stepwise search. Compared with the classical Sadek-Simiu method, the proposed stepwise search method achieves improved overall accuracy and applicability. The non-Gaussian features are found to be prominent at leading edge airflow separation on the crosswind side, the leeward corner cuts, the windward corner cuts, and the junction of two leeward surfaces at $45^{\circ}$ wind direction angle of square section. The junction of two leeward surfaces at $45^{\circ}$ wind direction angle exhibits stronger non-Gaussian features than the crosswind surface at $0^{\circ}$ wind direction angle. By giving the identical guarantee rate, the peak factors tend to be much larger in the regions with strong non-Gaussian properties and vice versa.
\end{abstract}

\section{Introduction}

Wind load is a decisive load in the design of high-rise buildings, especially for claddings, and most of the critical wind pressures on the building cladding are non-Gaussian signals. Therefore, it is critical to determine the maximum (minimum) wind pressures for design with an appropriate guarantee rate in the engineering design of high-rise building that considers both safety and economy.

For the wind-resistant design of buildings claddings, the distribution of extreme wind pressure is primarily considered, by which the design wind load with a certain guarantee rate can be determined based on reliability theory $[1,2]$. The object of extreme wind pressure estimation can be the parent distribution or the extracted extreme value distribution. When focusing on the parent distribution, the analysis will be carried out with the help of the Hermite polynomial transformation from Gaussian distribution to non-Gaussian distribution [3] and the cumulative probability function $[4,5]$. The main representatives are the peak factor method [6] proposed by Davenport in 1964 and the follow-up revised methods $[7,8]$. When the estimation takes the extreme value distribution as an object, it will mainly discuss the distribution model of wind pressure extremum, the parameter estimation method, the extremum sample acquisition method, and so on. The main representatives are the methods based on the Gumbel statistical model and the method based on the generalized extreme value distribution 
$(\mathrm{GEV})$. Since the extreme wind pressure distribution is much more related to the tail shape of the parent wind pressure distribution rather than its central shape, the estimation based on the parent distribution may be less appropriate for non-Gaussian signals with irregular tail shapes in their distributions. To further improve the estimation accuracy, many efforts have been made to improve the peak factor estimation method. Based on the Davenport method for the Gaussian process, several scholars have proposed different improvements to the calculation model and determination method of the non-Gaussian peak factor of fluctuating wind pressure. Kareem and Zhao [3] expressed the non-Gaussian process as Hermite polynomials of the Gaussian process considering higher-order statistics, thus extending the peak factor method to the non-Gaussian process. The process conversion method of Sadek-Simiu [4] uses the principle of equivalent probability to transform non-Gaussian process into Gaussian process and proposes a method for calculating the extreme value of non-Gaussian process. For the extreme value sequence, the Method of Independent Storms (MIS) proposed by Cook [9] extracts the data segments whose wind speed (or wind pressure) continuously exceeds the threshold value as an independent storm and takes the peak value of each independent storm as the sample of extreme value analysis. It should be noted that the segmentation method for extracting the extreme value is critical for the following estimation, and the shortest time delay of autocorrelation coefficient dropping from 1 to near 0 [10] or crossing zero for the first time [11] can be used to determine the optimum observation time interval. Zhao and $\mathrm{Gu}$ [12] applied the r-LOS model to the estimation of extreme wind pressure and verified it with wind tunnel test data. Quan and Gu Ming [13] segmented a single standard sample and then estimated the non-Gaussian wind pressure extreme value based on the classical extreme value theory.

Targeting at the wind pressure analysis of high-rise buildings, in recent research studies, many wind load calculation $[14,15]$ and wind pressure extremum estimation [16] methods are proposed. Huang and Chen [17] studied the wind load effects and equivalent static wind loads (ESWLs) of tall buildings based on measured synchronous surface pressures in a wind tunnel and proposed the practical methodology of modelling equivalent static wind loads (ESWLs) on tall buildings involving the influence of higher mode contributions. Especially, the nonGaussian characteristics of wind pressure fluctuation [18] and wind-induced response reliability [19] of high-rise buildings are analyzed. Based on investigation of 10 super high-rise building models (including rectangle, square, triangle, and Y-shape), Gu and Ye $[20,21]$ found that the wind pressure coefficients on the rectangular and square cross-section are largest in several shapes cross-sections. Tang [22] designed 11 models with rectangular, regular polygon, parallelogram, and other cross-section shapes, carried out a synchronous pressure test on them, studied the wind torque of building base, and fitted the closed formula of wind load power spectrum of the rectangular building. Ohkuma [23] and Choi [24] put forward the empirical formula of crosswind load power spectrum of high-rise buildings with rectangular sections and different length-width ratios and aspect ratios.

In 1970, Wardlaw and Moss [25] put forward the standard model of rectangular high-rise buildings (Commonwealth Advisory Aeronautical Research Council, CAARC). Therefore, scholars embarked on studies of wind pressure and the wind-induced response of CAARC model through pressure test, force test, aeroelastic test, and numerical calculation. Melbourne [26] compared measurement of surface pressures and response on the CAARC standard tall building model made at six establishments and found that the degree of agreement was good. Based on the CAARC model, Jamieson et al. [27] conducted wind tunnel tests on a rigid model of a rectangular shape high-rise building with rounded corners, concave corners, and chamfers and discussed the peak wind pressure of different arrangements of corner edges. The historical starting point of computational wind engineering (CWE) is that Smagorinsky [28] developed the Smagorinsky-Lilly model to Large Eddy Simulation (LES). The research works of wind tunnel testing and CWE are complementary. Huang et al. [29] simulated the wind load of CAARC based on the LES model and Reynolds Averaged Navier-Stokes (RANS) model, respectively, and compared with the test results. Elshaer et al. [30] and Huang et al. [31] gave the surface wind pressure distribution, base bending moment, and top displacement of the CAARC model under different wind directions through large eddy simulation (LES) and wind tunnel test, respectively, and compared the results with those of other wind tunnel laboratories. Tanaka and Lawen [32] obtained the wind pressure coefficient, fluctuating wind pressure spectrum, and curve of top displacement with wind direction angle at $2 / 3$ height of the CAARC model under different wind directions by pressure test and aeroelastic test. Dagnew and Bitsuamlak [33] investigated sheltering effects due to an adjacent CAARC model building placed in close proximity through LES and multiscale computational modeling approach in CFD. Alminhana et al. [34] proposed a numerical model based on the Taylor-Galerkin two-step scheme and the Finite Element Method to evaluate the aerodynamic performance of CAARC tall building models with different cross-section configurations. CFD is also applied to study the sensitivity of wind pressures over CAARC standard tall buildings to geometric and computational parameters [35] and to evaluate the upstream and downstream interference effect of two CAARC standard tall buildings [36]. To identify the influence of building shapes on coefficient distribution, taking oval-shaped high-rise buildings as examples, Zhao and He [37] numerically investigated the effects of height-width ratio (HWR) and height-thickness ratio (HTR) on mean wind pressure coefficients $(\mathrm{Cm})$ of building surfaces. Sometimes, the use of different unconventional shapes is also proved out to be efficient in reducing the total wind load and pressure of a tall building. The effect of wind on different unorthodox-shaped buildings has been studied such as '+' plan shaped [38], 'E' plan shaped [39], and ' $C$ ' plan shaped [40] tall building. Numerical as well as experimental analysis has been carried out to estimate wind loads with higher degree of confidence 
for these buildings with varying aspect ratios. Kim and Kanda [41] studied the aerodynamic force and wind pressure acting on super high-rise buildings with square cross sections and analyzed wind force and wind-induce response for setback and tapered buildings with different tapering ratios. Furthermore, aerodynamic comparison among common, conventional, and uncommon structures was also reported [42].

In this study, a numerical method for calculating the peak factor of non-Gaussian wind pressure named the stepwise search method is proposed, where the optimal segmented capacity $n^{*}$ is determined by using the rescaled range analysis method, and the independent extremum sequence is established with the help of the gradual independent segmentation method. Taking the wind pressures on claddings of the Shanghai World Financial Center as an example, the results of the proposed stepwise search method are compared with those of typical methods including the Sadek-Simiu method and the Kareem-Zhao method. Both the calculation of peak factor with a specified guarantee rate and the calculation of guarantee rate with a given peak factor have been carried out. The variation of peak factor with different wind direction angles and the correlation between peak factor and non-Gaussian features are then discussed. The characteristics and the error rates of mentioned above peak factor calculation methods are also given in this study.

\section{Stepwise Search Method for the Peak Factor of Non-Gaussian Wind Pressure Based on the Independent Extremum Subsequence}

\subsection{Stepwise Search Method for the Peak Factor of Non-} Gaussian Wind Pressure. As mentioned above, the windinduced pressures on high-rise building claddings are mostly non-Gaussian, but there is still a one-to-one correspondence between guarantee rate and peak factor. To determine the peak factor with a specified guarantee rate appropriately, a stepwise search method based on the bilateral guarantee rate model (see Figure 1) is proposed. The wind pressure extremum is determined according to a quantile corresponding to the specified guarantee rate. The guarantee rate corresponding to a quantile between the positive and negative extreme wind pressure coefficients symmetrical to the mean value is $P_{\text {fix }}$. It is defined as follows:

$$
\begin{aligned}
P\left\{\hat{C}_{P}\right\} & -P\left\{\stackrel{\vee}{C}_{P}\right\}=P_{\text {fix }}, \\
f(k)= & P\left\{C_{P \text { mean }}+k \cdot C_{P \text { rms }}\right\} \\
& -P\left\{C_{P \text { mean }}-k \cdot C_{P \text { rms }}\right\}-P_{\text {fix }}=0,
\end{aligned}
$$

where $k$ is the peak factor, while $C_{P \text { mean }}$ and $C_{P \text { rms }}$ are the mean pressure coefficient and root mean square of the fluctuating pressure coefficient, respectively. To solve this equation and obtain the peak factor $k$, the stepwise search method is used. Assuming that the unknown $k=x$, the idea is to divide the solution interval into many equal subinterval and search for a smaller subinterval step by step. Specifically, the algorithm starts from the beginning of the interval with $x=a$ and moves to the end step by step according to the selected step length $h$, during which the sign of $f(x)$ is checked at each step. If the sign of $f(x)$ changes, the solution must be on the corresponding interval $\left[x_{i}, x_{i+1}\right]$. Therefore, by repeating this process on the subinterval $\left[x_{i}, x_{i+1}\right]$, the interval of the solution can be quickly narrowed down (see Figure 2).

By using the stepwise search method, the peak factor of non-Gaussian wind pressure with a certain guarantee rate can be calculated based on reliability theory. In this study, the extreme value distribution is selected as the research object to reduce the influence of non-Gaussian characteristics. Even so, it is difficult to fit accurately the wind pressure coefficients with strong non-Gaussian features by using a settled probability distribution function, but the numerical method in the proposed process avoids this disadvantage, which makes it highly adaptable to any non-Gaussian distribution.

\subsection{Rescaled Range Analysis-Based Optimum Segmentation} Capacity $n^{*}$. Extreme value theory should be built on the premise of independent and identical distribution. Before estimating the peak factor by the stepwise search method, the independent extremum subsequence must be separated from the parent wind pressure time history. The rescaled range analysis method is thus introduced to determine the optimal segmentation capacity $n^{*}$. Combined with the gradual independent segmentation method described in Section 2.3, the independence of the extreme values can be ensured by making the data interval between adjacent extreme values greater than $n^{*}$.

The rescaled range analysis method is used to detect the long-term memory of wind pressure time history, determine the vanishing point of long-term memory behavior, and take the node as the sectional time to ensure the independence of the sectional time history. The rescaled range analysis method was first proposed by Hurst, a British scholar, in a study of the Nile water conservancy project. In the study, the range $R$ was removed by the standard deviation $S$ of the observed value to obtain a dimensionless ratio called the $R / S$ statistic. Suppose $C=\left\{c_{1}, c_{2}, \ldots, c_{N}\right\}$ is the wind pressure time history, with a data length $N$ and a corresponding time $T$. Then, $C$ is divided into $m=T / t$ segments $C=\left\{C^{(1)}, C^{(2)}, \ldots, C^{(m)}\right\}$, and the capacity of each segment $C^{(k)}(n)=\left\{c_{(k-1) \cdot n+1}, \ldots, c_{k \cdot n}\right\}, \quad k=1,2, \ldots, m, \quad$ is $n=N / m$. For the wind pressure time history $C$, when the segment length is $n$, the calculation process of the statistic $R / S(n)$ is given as follows.

The average value $E^{(k)}(n)$ of the $k^{\text {th }}$ segment is

$$
E^{(k)}(n)=\frac{1}{n} \sum_{i=1}^{n} c_{(k-1) \cdot n+i}, \quad k=1,2, \ldots, m .
$$

The accumulated deviation $Y^{(k)}(n)$ of the $k^{\text {th }}$ segment is

$Y^{(k)}(n)=\sum_{i=1}^{n}\left(c_{(k-1) \cdot n+i}-E^{(k)}(n)\right), \quad k=1,2, \ldots, m$.

The range $R^{(k)}(n)$ of the $k^{\text {th }}$ segment is 


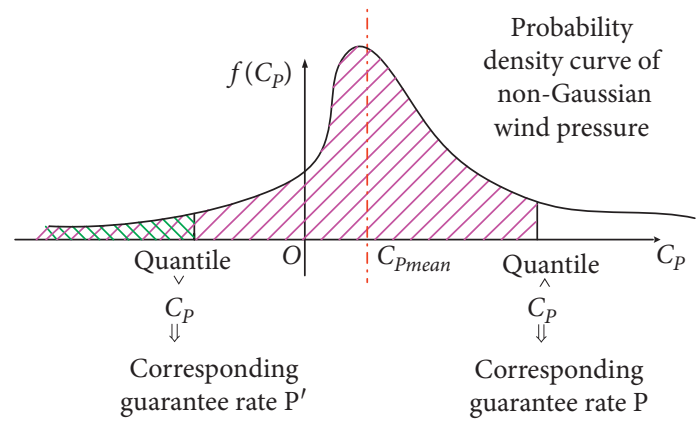

Figure 1: Bilateral guarantee rate model.

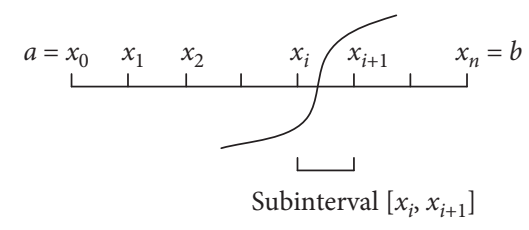

FIgURE 2: Stepwise search method sketch.

$R^{(k)}(n)=\max \left(Y^{(k)}(n)\right)-\min \left(Y^{(k)}(n)\right), \quad k=1,2, \ldots, m$

The standard deviation $S^{(k)}(n)$ of the $k^{\text {th }}$ segment is

$S^{(k)}(n)=\sqrt{\frac{1}{n} \sum_{i=1}^{n}}\left[\left(c_{(k-1) \cdot n+i}-E^{(k)}(n)\right)\right]^{2}, \quad k=1,2, \ldots, m$.

The statistic $R / S^{(k)}(n)$ of the $k^{\text {th }}$ segment is

$$
\frac{R^{(k)}(n)}{S^{(k)}(n)}
$$

When the segment length is $n$, the statistic $R / S(n)$ of the wind pressure coefficient time history $C$ is

$$
\frac{R}{S(n)}=\frac{1}{m} \sum_{k=1}^{m} \frac{R}{S^{(k)}(n)}
$$

The formula for the $Q$ statistic is $Q(n)=\ln [(R / S)(n)]$, which can be used to determine whether the time sequence has nonperiodic cycles and then determine the average cycle length, that is, the past trend affects the future time length. For the time series of independent random processes, the curve of $Q(n) \sim \ln n$ is a straight line. If the time series is random, the curve of $Q(n)$ statistic should be a horizontal line, while a downward or upward incline of the curve shows that it is antipersistent or persistent, respectively. The critical point of the segment length (i.e., the sample capacity $n^{*}$ ) is then determined when the curve of $Q(n)$ changes markedly at a point such that its increasing trend stops, which indicates that the long-term memory of the stochastic process disappears [43, 44]. Therefore, the time history of wind pressure coefficient is divided into segments with length $n^{*}$.
2.3. Gradual Independent Segmentation Method for Independent Extremum Sequences. The wind pressure time history can be divided into several segments according to the optimal sectional capacity $n^{*}$, and the extremum can be selected from each segment to form the extremum sequence. Because the location of the extreme value in each segment is random, the interval between two adjacent extreme values may be smaller than the optimal segment capacity $n^{*}$, which indicates that the statistical independence of the extracted values cannot be theoretically guaranteed. Therefore, the gradual independent segmentation method is proposed to solve the problem.

The idea of the gradual independent segmentation method is derived from the time interval method $[45,46]$ in statistics. In the time interval method, the independence of the data is judged by the time interval before and after the threshold. On this basis, the gradual independent segmentation method should meet the following conditions: (1) the interval between each pair of adjacent extremums should be greater than the optimal segment capacity $n^{*}[47],(2)$ the extreme value $c_{k}(k=1,2, \ldots, m)$ should be the maximum (or minimum) value of each segment, (3) the maximum value of the first segment with length $n^{*}$ and its previous data should be removed to avoid the influence of incomplete vortex shedding at the beginning of the data sample, and (4) if the above conditions are met, the interval between extremums should be set as small as possible to keep more detailed information of the original data and reflect more accurate characteristics of the wind pressure.

Specifically, for a wind pressure time history $C(j)(j=$ $1,2, \ldots, N)$ with sample capacity $N$ and optimal segment capacity $n^{*}$, the independent extremum sequence is selected as follows (see Figure 3). (1) Find the first extreme value $c_{0}$ of a segment with length $n^{*}$ and then remove it with the previous data to avoid the influence of incomplete vortex shedding at the beginning of the data sample. Suppose the position of $c_{0}$ is $n_{0}$, and the first extreme value $c_{1}$ can then be selected from the segment with length $n^{*}$ starting from the point $n_{0}+1$. (2) To obtain the $k^{\text {th }}$ extreme value $c_{k}$, two segments with length $n^{*}$ are considered from the position of $c_{k-1}$. The first number found in the second segment with length $n^{*}$ greater than or equal to the maximum (absolute value of minimum) number of the first segment with length $n^{*}$ is $c_{k}$. If all numbers of the second segment are smaller than the maximum (absolute value of minimum) number of the first segment, the maximum (absolute value of minimum) number of the first segment will replace $c_{k-1}$. According to the new $c_{k-1}$, this step can start over with the updated two segments. (3) Repeat step (2) until the end of the time history, and then, the independent extremum sequence can be obtained.

In Figure $3, c_{k}$ is the $k^{\text {th }}$ extreme value, $n_{k}$ is the interval from the $(k-1)^{t h}$ extreme value $c_{k-1}$ to the $k^{\text {th }}$ extreme value $c_{k}$, and $n_{k} \geq n^{*}+1,(k=1,2, \ldots)$.

Therefore, a numerical statistical approach for peak factor of non-Gaussian wind pressure has been proposed and is referred to as the stepwise search method. The flowchart of this method is shown in Figure 4. 


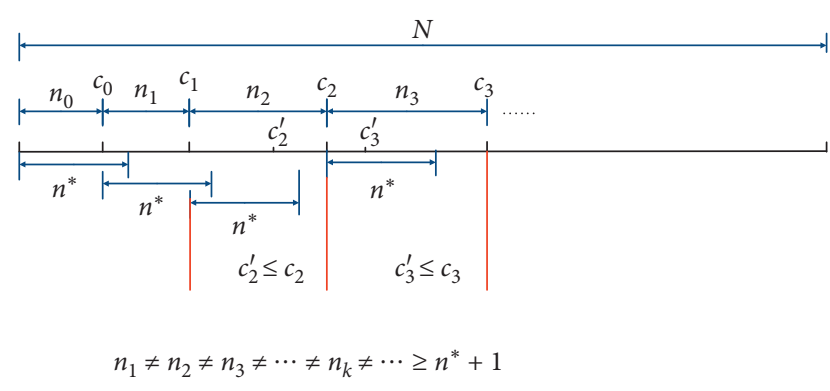

FIGURE 3: Gradual independent piecewise method sketch map.

\section{Research Methods Based on Wind Tunnel Test}

3.1. Wind Field Simulation. This study takes the Shanghai World Financial Center as an engineering background, and the pressure tests were carried out on a rigid model in the TJ2 Wind Tunnel at Tongji University. The TJ-2 Wind Tunnel is a boundary layer tunnel of closed-circuit-type, and the working section of the tunnel is $3 \mathrm{~m}$ wide, $2.5 \mathrm{~m}$ high, and $15 \mathrm{~m}$ long. The achievable mean wind speed ranges from $0.5 \mathrm{~m} / \mathrm{s}$ to $68.0 \mathrm{~m} / \mathrm{s}$, adjustable continuously. The mean wind speed profile together with the turbulence intensity for terrain category $\mathrm{C}$ was simulated mainly by using hybrid passive devices including roughness blocks, spirelets, and vertical-bar fences. The simulation result and the theoretical formula according to the load code for the design of building structures by Ministry of Construction of the People's Republic of China (GB50009 - 2001) are shown in Figure 5.

3.2. Test Model. The rigid model for wind tunnel pressure test is shown in Figure 6, together with the definitions of model orientation, wind direction angle, and coordinate axis of this rigid model. Each pressure signal was sampled with 6000 data points with a frequency of $312.5 \mathrm{~Hz}$, which means the sampling time is $19.2 \mathrm{~s}$. Because of the limitations of the channels of the electronic scan valve, the signals of measuring taps could not be collected simultaneously at a time and should be divided into 4 groups of A, B, C, and D. The way of grouping situation, the characteristic size of model section, and the layout of surface pressure measurement taps are shown in Figure 7. It can be seen that pressure measurement taps were distributed on 22 levels, which were numbered from bottom to top of the model. There are 28 to 40 measurement taps in each level, changing with height. In this study, ' $L-i$ ' is used to indicate the measurement taps, where $L$ represents the number of levels and $i$ represents the measurement point number at this level. For example, '5-17' represents the 17 measurement point of the $5^{\text {th }}$ level.

3.3. Test Conditions. Corresponding to the $1: 350$ length scales, the total height of this model is $1.4 \mathrm{~m}$. The side elevation width of building model changes from $230.5 \mathrm{~cm}$ to $27.09 \mathrm{~cm}$ from bottom to top. In this test, the reference wind speed was $14 \mathrm{~m} / \mathrm{s}$, which was monitored by a Pitot tube at $1.2 \mathrm{~m}$ height in wind tunnel (above the gradient height, equivalent to the actual $420 \mathrm{~m}$ height), about $1.0 \mathrm{~m}$ away from the side wall of the wind tunnel. Considering a recurrence period of 50 years, the actual wind speed corresponding to the reference point is $52.93 \mathrm{~m} / \mathrm{s}$ according to the conversion of basic wind pressure of the C-type landform in Shanghai; then, the wind velocity ratio can be deduced to be $\lambda_{V}=0.2645$. The time ratio is $\lambda_{t}=\lambda_{L} / \lambda_{V}=0.0108$, which can be deduced by the dimensional analysis method.

\section{Results and Parametric Studies}

4.1. Validation and Comparison of Peak Factors. In this study, the numerical method of "stepwise search" is used to overcome the shortcomings of setting up a specific probability distribution model by the conventional analysis approach for non-Gaussian signals, thus making the proposed method more adaptable to the complex estimation of peak factors for non-Gaussian wind pressures. For comparison, the peak factors of wind pressures were calculated by the stepwise search method proposed in this study and by the other two approaches suggested by Kareem and Zhao and Sadek and Simiu. To compare the performances of the three methods mentioned above, their results are compared with the peak factor calculated directly from the observed average extremum in this study.

4.1.1. Variation of Peak Factor with Wind Direction Angle. Due to the complexity of the architectural shape in this study, only measurement point 5-17 was selected as an example to describe the main trend of the wind pressures on the claddings, which are summarized as follows: (1) the crosssection of the $5^{\text {th }}$ measurement level $(212 \mathrm{~mm}$, which corresponds to a real building height of $74.5 \mathrm{~m}$ ) is nearly quadrilateral and (2) the location of measurement point 5-17 is in Figure 8, which shows the cross section of the $5^{\text {th }}$ measurement level and the definition of wind direction angle. For the $90^{\circ}$ wind direction, it is located at the leading edge of airflow separation on the side face, while for the $225^{\circ}$ wind direction, it is located at the center of the wake flow.

To compare the peak factors calculated by different methods, the error rate of the peak factor is defined based on the observed value as follows:

$$
\varepsilon_{i}=\frac{k^{m}(i)-k^{0}(i)}{k^{0}(i)}
$$

where $k^{m}(i)$ is the peak factor of measurement point $i$ calculated by method $m$ and $k^{0}(i)$ is the observation peak factor of measurement point $i$. Equation (8) is generally used for a single measurement point, and the average error rate is used to evaluate the overall error rate of multiple measurement points. The average error rate is defined as the root mean square of the single error rates shown in

$$
\bar{\varepsilon}=\sqrt{\frac{1}{n} \sum_{j=1}^{n} \varepsilon_{j}^{2} .}
$$


(1) Obtain non-Gaussian wind pressure from wind tunnel tests
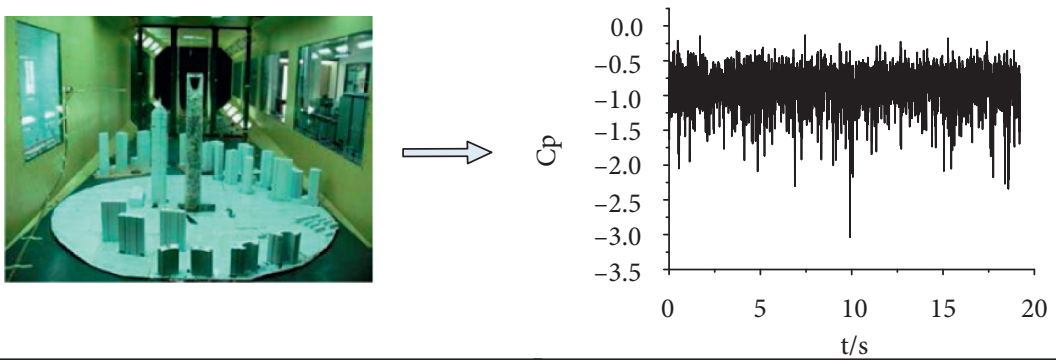

dI

(2) Determine the optimal segmenting capacity $n^{*}$ by using the "rescaled range analysis method" Based on the bilateral guarantee rate model.

Statistics: $\quad Q(n)=\operatorname{In}[(R / S)(n)] \quad R / S(n)=1 / m \sum_{\mathrm{k}=1}^{m} R / S^{(k)}(n)$

$N$

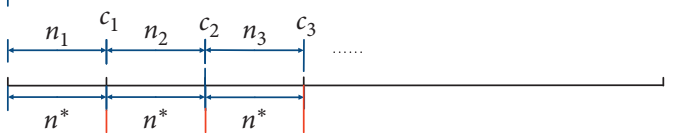

$n_{1}=n_{2}=n_{3}=\cdots=n_{k}=\cdots=n^{*}$

th

(3) Extract independent extremum sequence with the help of gradual independent segmentation method

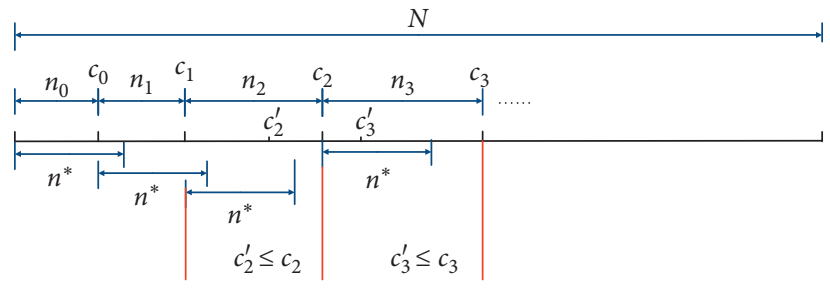

$n_{1} \neq n_{2} \neq n_{3} \neq \cdots \neq n_{k} \neq \cdots \geq n^{\prime}+1$

لֶ

(4) Caliculate the peak factor for a specified guarantee rate by the "stepwise scarch method"

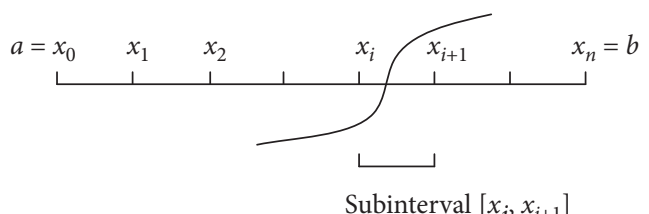

$$
P\left\{\hat{C}_{P}\right\}-P\left\{\check{C}_{P}\right\}=P_{f i x}
$$

$f(k)=P\left\{C_{\text {Pmean }}+k \cdot C_{\text {Prms }}\right\}-P\left\{C_{\text {pmean }}-k \cdot C_{\operatorname{Pr} m s}\right\}-P_{\text {fix }}=0$

FIGURE 4: Flowchart of stepwise search method.

Based on reliability theory, the peak factor with a certain guarantee rate can be found by the stepwise search method, so an appropriate guarantee rate should be determined firstly in this study. The peak factor for Gaussian distribution is thus adopted as a reference, and several typical peak factors of the Gaussian model and their corresponding unilateral and bilateral guarantee rates are listed in Table 1, where the relationship between the unilateral and bilateral 


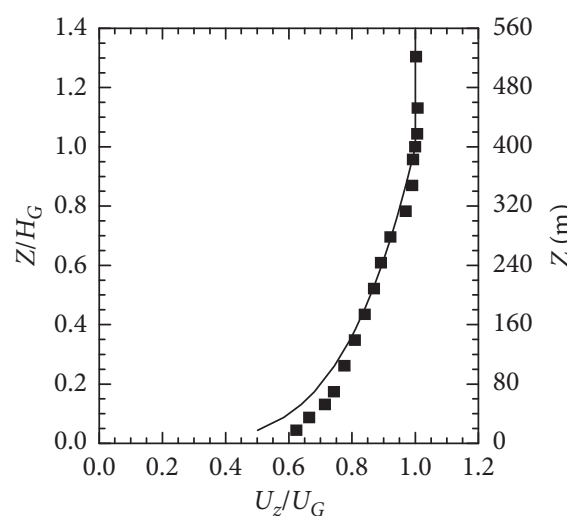

- Results of test Results of formula: $U_{z} / U_{G}=\left(Z / H_{G}\right)^{0.22}$

(a)

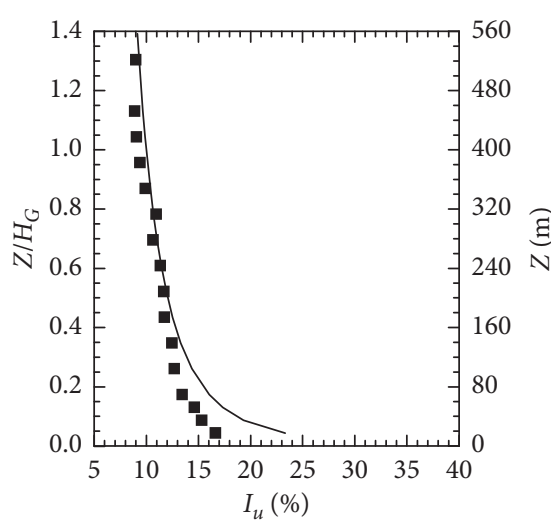

- Results of test

Results of formula: $I_{u}=0.1\left(Z / H_{G}\right)^{-0.22-0.05}$

(b)

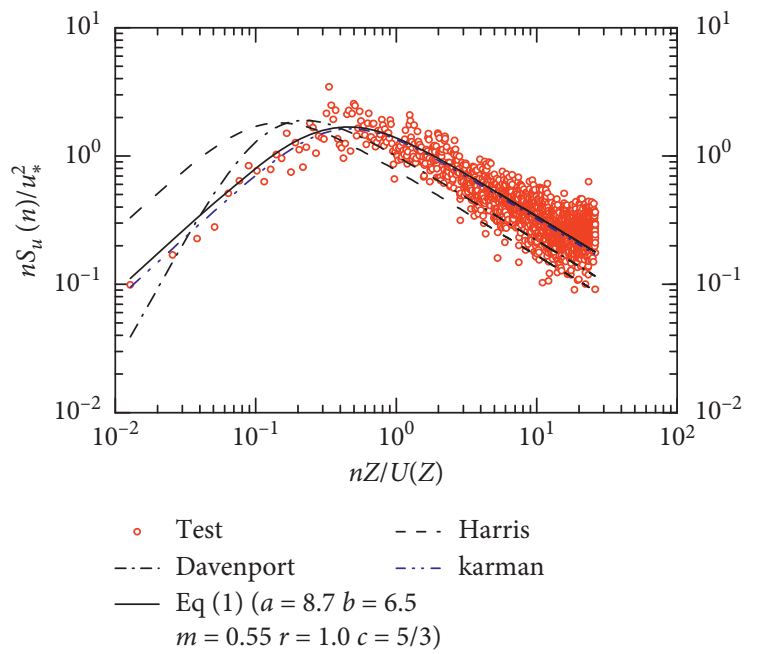

(c)

Figure 5: Wind field simulation. (a) Mean wind speed profile. (b) Turbulence profile of along-wind. (c) Fluctuating wind spectrum of alongwind.
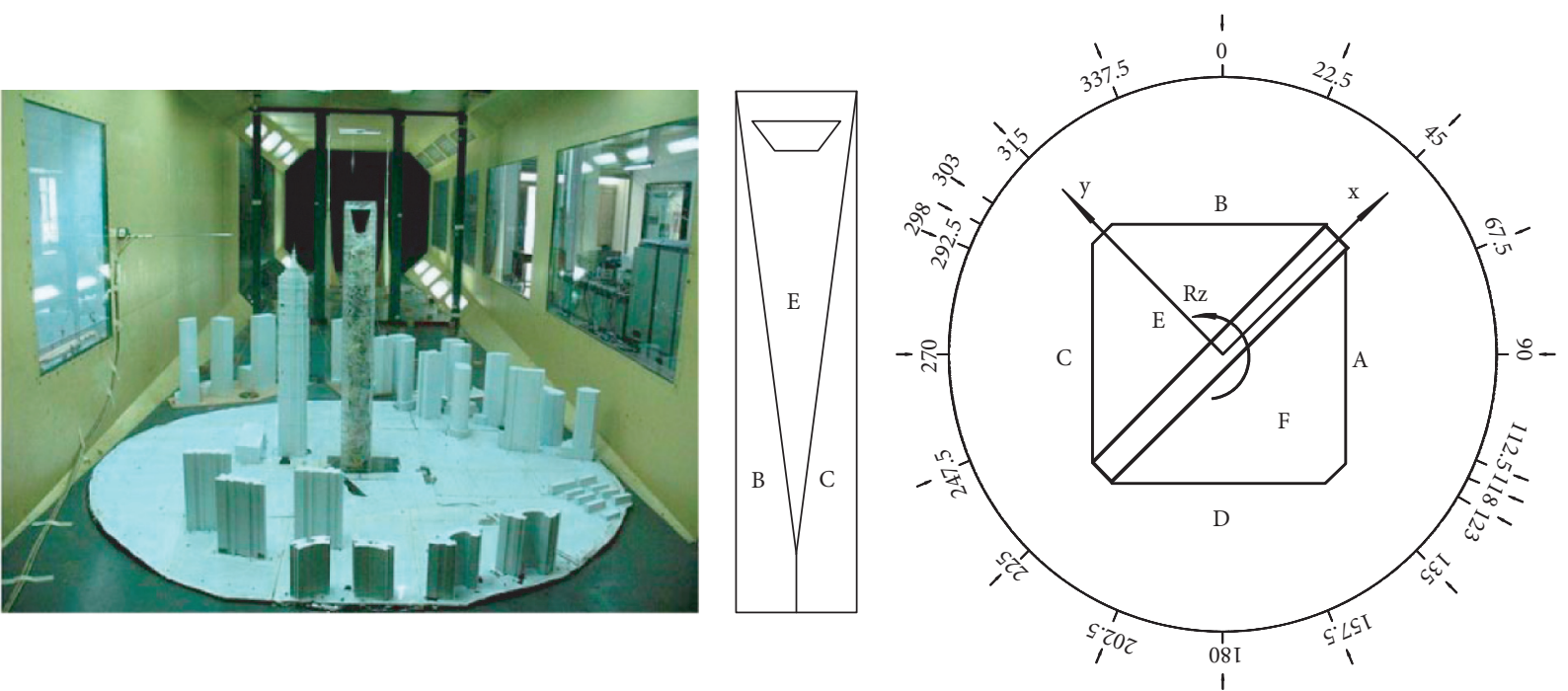

Figure 6: Setup, facade, wind direction, and coordinate axis of the rigid model. 


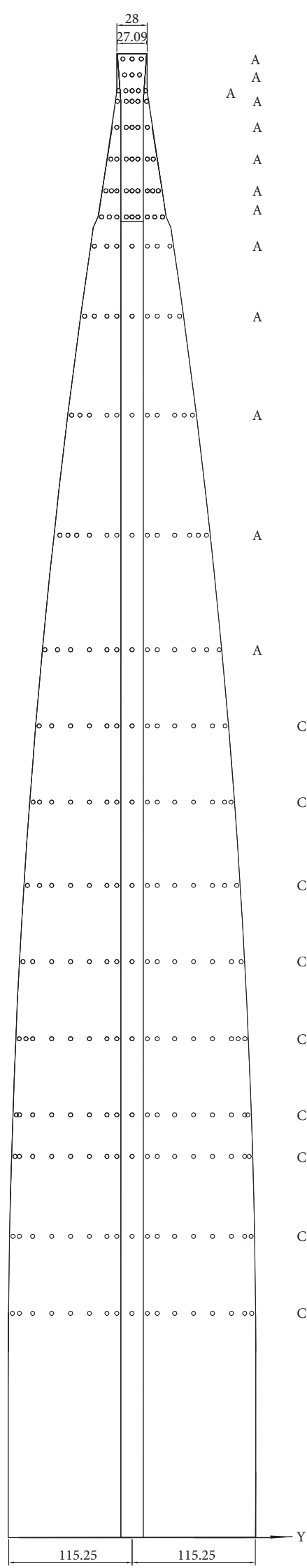

(a)

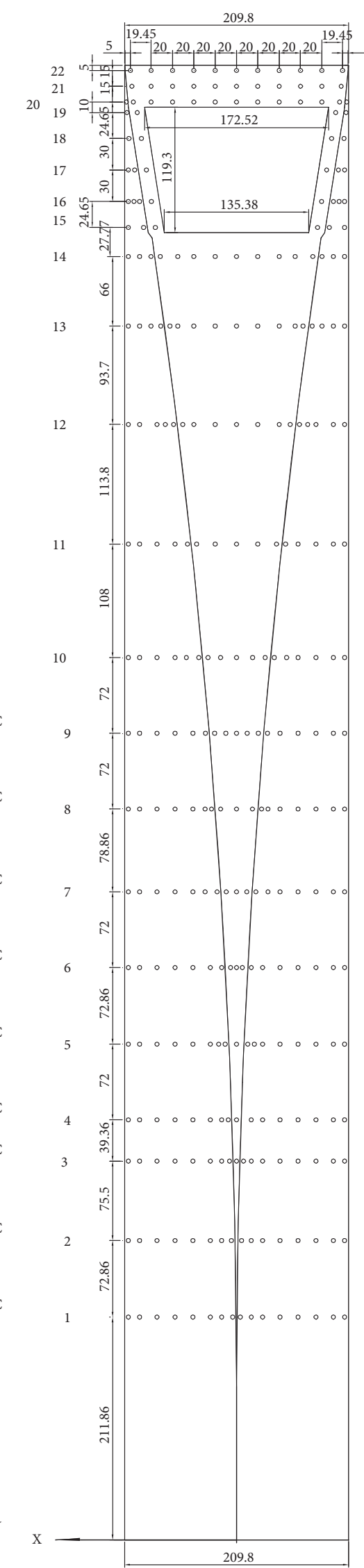

(b)
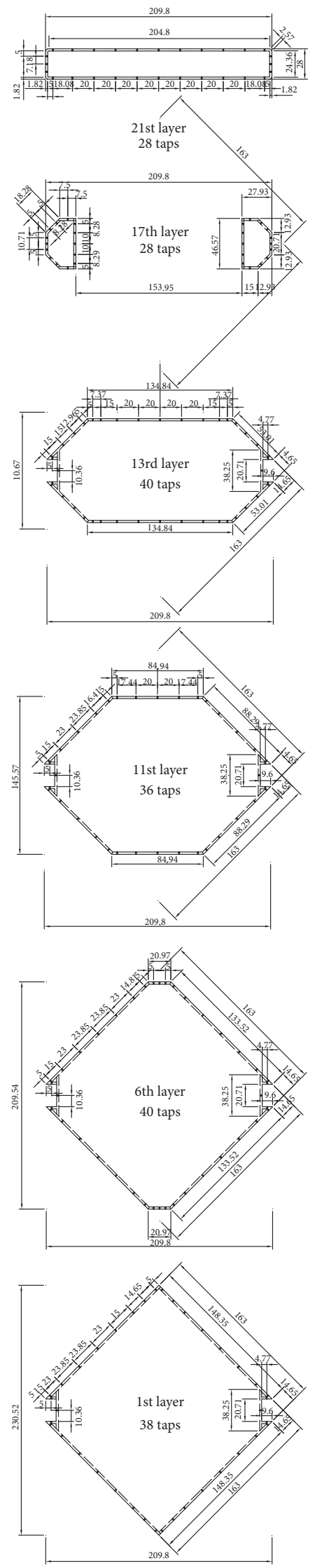

(c)

FIGURE 7: The shape and measuring tap arrangement of façade and cross-sections of typical heights. (a) Side elevation. (b) Front elevation. (c) Cross section on typical height. 


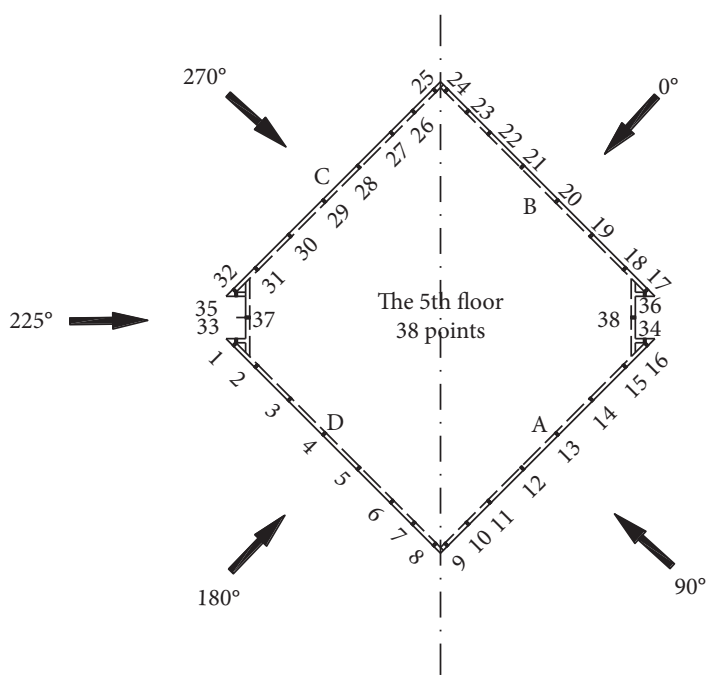

(a)

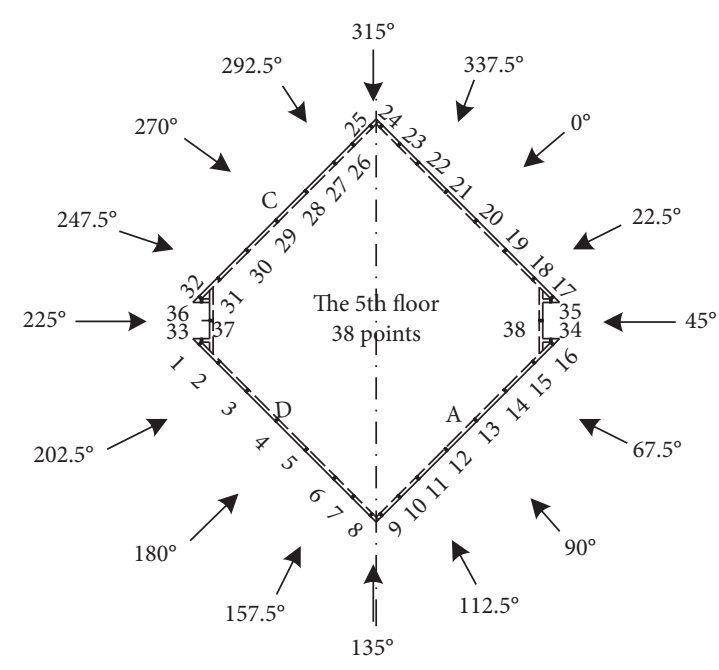

(b)

Figure 8: Cross section of the $5^{\text {th }}$ measurement level and definition of wind direction angle.

TABle 1: Peak factor of the Gaussian model and corresponding unilateral and bilateral guarantee rates.

\begin{tabular}{lccccccccc}
\hline \multirow{2}{*}{ Project } & \multicolumn{9}{c}{ Number } \\
& 1 & 2 & 3 & 4 & 5 & 6 & 7 & 8 & 9 \\
\hline Peak factor g & 1.645 & 2 & 2.2 & 2.3263 & 2.5 & 3 & 3.2649 & 3.3 & 3.5 \\
Corresponding unilateral guarantee rate P & 0.95 & 0.9772 & 0.9861 & 0.99 & 0.9938 & 0.9987 & 0.9995 & 0.9995 & 0.9998 \\
Corresponding bilateral guarantee rate P & 0.9 & 0.9544 & 0.9722 & 0.98 & 0.9876 & 0.9974 & 0.999 & 0.999 & 0.9996 \\
\hline
\end{tabular}

guarantee rates of the Gaussian model is $P_{\text {bilateral }}=2 P_{\text {unilateral }}-1$. In this study, the guarantee rate $P_{\text {bilateral }}=99.96 \%$ according to a peak factor of 3.5 is selected to be the threshold.

Figure 9 shows the variation of peak factors and error rates of each method with wind direction angle for measurement point 5-17. The figure shows that when the wind direction angle is within $0^{\circ} \sim 67.5^{\circ}$, measurement point $5-17$ is located on the windward side. Since the wind pressure in this case approximately obeys Gaussian distribution, the results of all three methods agree well with the observed values. When the wind direction angle is within $90^{\circ} \sim 315^{\circ}$ and measurement point $5-17$ is located on the side face and leeward face, the wind pressures no longer agree with the Gaussian distribution, and the peak factors calculated by the different methods range from 4.5 to 7.3. As shown in Figure 9(b), the stepwise search method attains the lowest error rate, and the error rate is positive, which indicates safer results.

There are three remarkable local peaks shown in Figure 9(a) (marked by red circles), where the calculated results of the peak factor method deviate far from the observed values due to strong non-Gaussian features. In the case of $90^{\circ}$ wind direction angle, measurement point 5-17 is located near the separation point on the cross-wind surface, and the values of the pressure time history in the vortex shedding area of the crosswind side are quite different from the mean value, and the negative pressure extreme value is larger, showing a strong non-Gaussian feature. In the case of $225^{\circ}$ wind direction angle, measurement point $5-17$ is located in the center of wake flow on the leeward side, where the negative pressure pulse occurs and changes quickly, which also makes the non-Gaussian characteristic prominent. Similarly, in the case of $180^{\circ}$ wind direction angle, measurement point 5-17 is located on the leeward side and is strongly affected by the wake flow. However, in the case of $225^{\circ}$ wind direction angle, measurement point $5-17$ is in the wake center, while in the case of $180^{\circ}$ wind direction angle, measurement point 5-17 is only affected by the wake flow; therefore, the non-Gaussian property in the former case is much stronger. A non-Gaussian distribution does not refer to a particular type of distribution but refers to all types of distributions except a Gaussian distribution. The third- and fourth-order statistics (skewness and kurtosis, respectively) are important parameters to distinguish Gaussian distributions from non-Gaussian distributions and important indicators to describe the probability performance of nonGaussian distributions. In this study, the wind pressures with absolute values of skewness greater than 0.2 or absolute values of kurtosis greater than 3.4 are defined as nonGaussian wind pressures [48]. Table 2 lists the non-Gaussian judgement of measurement point 5-17 under different wind direction angles. It can be seen that most wind pressures on the claddings of this high-rise building are non-Gaussian under the interference of wake flow induced by surrounding buildings and the complex body itself. 


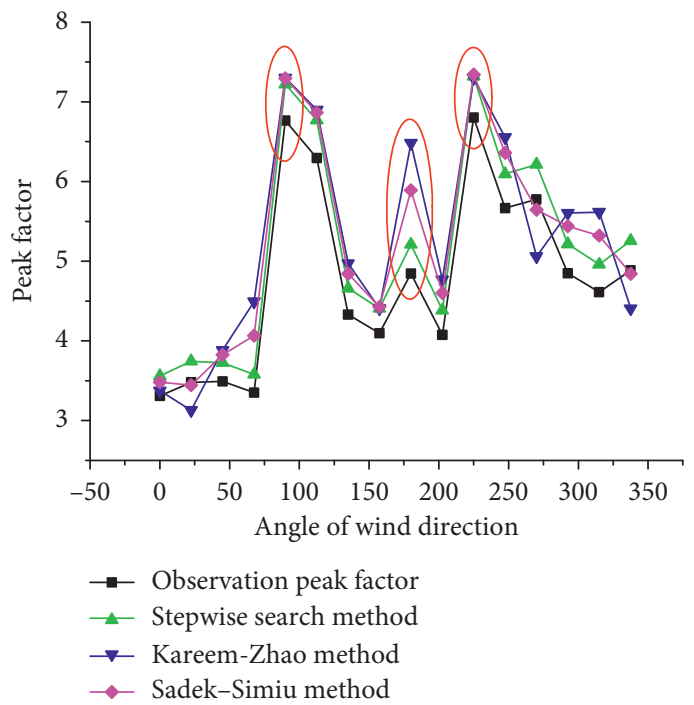

(a)

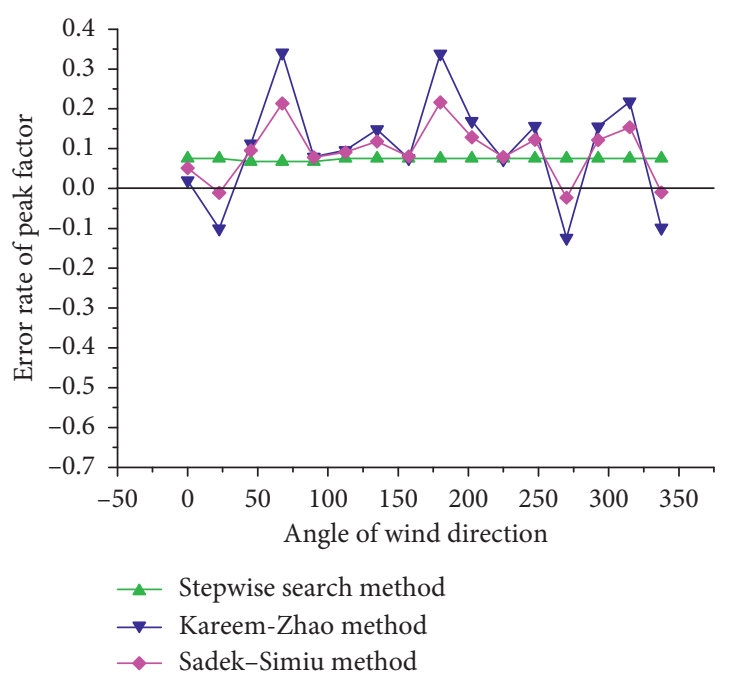

(b)

Figure 9: (a) Variation of peak factor with wind direction angle and (b) variation of error rate with wind direction angle of measurement point 5-17.

TABle 2: Non-Gaussian judgement of measurement point 5-17 under different wind direction angles.

\begin{tabular}{|c|c|c|c|c|}
\hline Measurement point & Angle & Skewness & Kurtosis & Judgment value of non-Gaussian character \\
\hline \multirow{16}{*}{$5-17$} & 0 & -0.1612 & 2.8153 & 0 \\
\hline & 22.5 & -0.1777 & 2.5974 & 0 \\
\hline & 45 & -0.3736 & 2.9576 & 0 \\
\hline & 67.5 & -0.7848 & 3.2996 & 1 \\
\hline & 90 & -2.994 & 13.1109 & 1 \\
\hline & 112.5 & -1.7357 & 7.6009 & 1 \\
\hline & 135 & -0.9562 & 3.9364 & 1 \\
\hline & 157.5 & -0.5509 & 3.2805 & 1 \\
\hline & 180 & -1.286 & 6.3679 & 1 \\
\hline & 202.5 & -0.5948 & 3.9574 & 1 \\
\hline & 225 & -3.6062 & 16.4207 & 1 \\
\hline & 247.5 & -1.3952 & 6.353 & 1 \\
\hline & 270 & -0.5395 & 4.2028 & 1 \\
\hline & 292.5 & -1.0439 & 4.6158 & 1 \\
\hline & 315 & -1.147 & 4.6066 & 1 \\
\hline & 337.5 & -0.3999 & 3.7862 & 1 \\
\hline
\end{tabular}

(Notes: 0 represents Gaussian or near Gaussian, and 1 represents non-Gaussian. See Figure 8(b), for the angle).

4.1.2. Comparison of Peak Factors Calculated by Using the Different Models. To compare the peak factors calculated by using the different calculation models, the $5^{\text {th }}$ measurement level at $90^{\circ}$ wind direction angle is selected as an example in this subsection; therefore, the comparison between the calculated and observed peak factors is shown in Figure 10. Generally, the estimation values of the stepwise search method, the Kareem-Zhao method, and the Sadek-Simiu method are larger than the observed values, and the results of the stepwise search method in this study are the closest to the observation values. For most of the measurement points in the non-Gaussian region of the cross-wind surface, the peak factors calculated by the stepwise search method and Sadek-Simiu method are closer to the observed value than those calculated by the other method. The peak factor estimated by the stepwise search method based on reliability theory is better than the expected peak factor obtained by the Sadek-Simiu method. The peak factors of most of the measurement points are overestimated by the Kareem-Zhao method, which are equivalent to the upper envelope of the peak factors. One reason for this inaccuracy is that the Hermite polynomial transformation model from Gaussian space to non-Gaussian space has errors. The other reason is that the estimation method based on parent distribution is affected by the sampling uncertainty, which increases with increasing statistical order. Therefore, the more statistical order is considered, the more inaccurate the estimation results will be.

The peak factors of the measurement points (1 8, 17 24), which are located at the cross-wind surface, and of the measurement points (24-32), which are located at the leeward surface, are larger than those of the measurement 


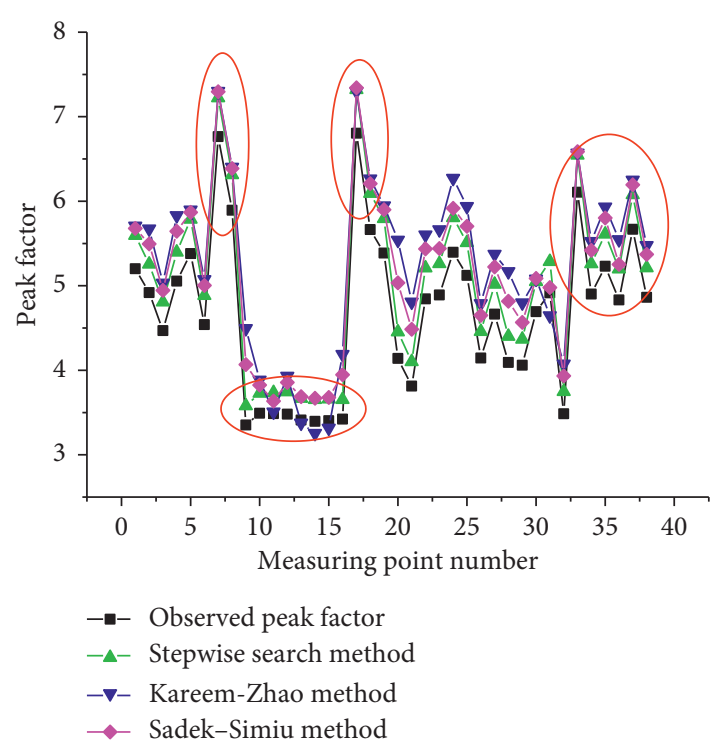

Figure 10: Comparison of peak factors at the $5^{\text {th }}$ measurement level with $90^{\circ}$ wind direction angle.

points (9-16), which are located at the windward side in the Gaussian region. The reason for these results is that there is a complex wake center on the leeward side affected by the wake flow, where the negative pressure pulse has a large negative bias extreme value and it changes rapidly; thus, the nonGaussian characteristics are significant. The wind pressures on the crosswind surface are strongly non-Gaussian due to the separation, reattachment, and vortex shedding of the incoming flow. For the windward side, the angle between the wind direction and the normal line of the cladding decreases, the wind area of the measurement point increases, the wind pressure effect is uniformly equalized from the surroundings to the measurement point, and the negative pressure extreme value decreases, exhibiting Gaussian or near Gaussian. Measurement points 33, 35, and 37 are located at the leeward side corner cuts, where the shedding eddy rolls along the side face to form a wake area; thus, the skewness and kurtosis change over a large span and the negative pressure pulse is prominent; thus, the non-Gaussian characteristic is significant. Measurement points 34, 36, and 38 are located at the windward corner cuts, forming a pocket; therefore, a squeezing effect appears due to incoming and follow-up flow thus causing a high-speed vortex, so the non-Gaussian feature is also obvious in this area.

Figure 11 shows the deviation of the peak factors from the observed values for all 808 measurement points with the three methods. Compared with other methods, the peak factors calculated by the proposed stepwise search method fit the observation peak factors with the highest goodness-of-fit $R^{2}$. Table 3 lists the average error rates of the peak factors calculated by different calculation methods under different working conditions. Generally, the peak factor errors calculated by the stepwise search method are smaller than those of other methods. The results of the stepwise search method are close to the observed values for different samples, with a small degree of dispersion.
As shown in Table 3, the average error rates of the peak factor calculated by different methods at $45^{\circ}$ wind direction angle are generally larger than those at other wind direction angles because the two windward faces are in $+45^{\circ}$ and $-45^{\circ}$, respectively, which makes the wind receiving condition of the measurement points on the two claddings nonuniform relative to $0^{\circ}$ wind direction angle, and the negative pressure extreme value increases. Concurrently, the separation angle between the incoming flow and the two leeward surfaces is approximately $45^{\circ}$, and the two leeward surfaces are in $-135^{\circ}$ and $135^{\circ}$, respectively. The $135^{\circ}$ leeward surface exhibits stronger non-Gaussian features than the cross-wind surface. This is because the included angle between the $135^{\circ}$ leeward surface and the incoming flow better meets the movement trend of the incoming flow separation, the vortex shedding, and reattachment and makes the consistency of the vortices motion stronger on the $135^{\circ}$ leeward surface, so the nonGaussian character is stronger. The average error rate calculated by the stepwise search method is controlled to be within $10 \%$, while the average error rate of the peak factor calculated by other methods remains within $20 \%$.

\subsection{Conversion between the Guarantee Rate and Peak Factor}

\subsubsection{Calculation of Peak Factor with Specified Guarantee} Rate. With the help of stepwise search method, the peak factor corresponding to a specified guarantee rate can be calculated quickly for any non-Gaussian wind pressure. The peak factors corresponding to $99 \%$ guarantee rate of measurement points on the $5^{\text {th }}$ measurement level at $90^{\circ}$ wind direction angle (see Figure 8) are calculated by the stepwise search method and listed in Table 4, which have been classified as the windward side (Face A), the crosswind side (Face D and Face B), and the leeward side (Face C). The results show that the peak factors corresponding to $99 \%$ guarantee rate are generally greater than 3.5 , which can provide a $99.96 \%$ guarantee rate in the case of Gaussian distribution, while the peak factor corresponding to $99 \%$ guarantee rate is 2.3263 for Gaussian distribution (see Table 1). The peak factors of measurement points at the windward surface range from 3.38 to 3.53 , with an average value of 3.47. The peak factors of measurement points at the crosswind surface range from 3.87 to 7.31 , with an average value of 5.29. The peak factors of measurement points at the leeward surface range from 3.54 to 5.2, with an average value of 4.46. This shows that the traditional peak factor given based on Gaussian assumption might be unsafe for most of the working conditions, especially for those measurement points located in the incoming flow separation zone on the cross-wind surface and the wake center of the leeward surface. It should also be noted that even though most of the peak factors increase when the non-Gaussian character becomes stronger, this kind of relationship cannot be guaranteed.

4.2.2. Calculation of the Guarantee Rate with Specified Peak Factor. By the stepwise search method, the guarantee rate for a certain peak factor can also be obtained for any non- 


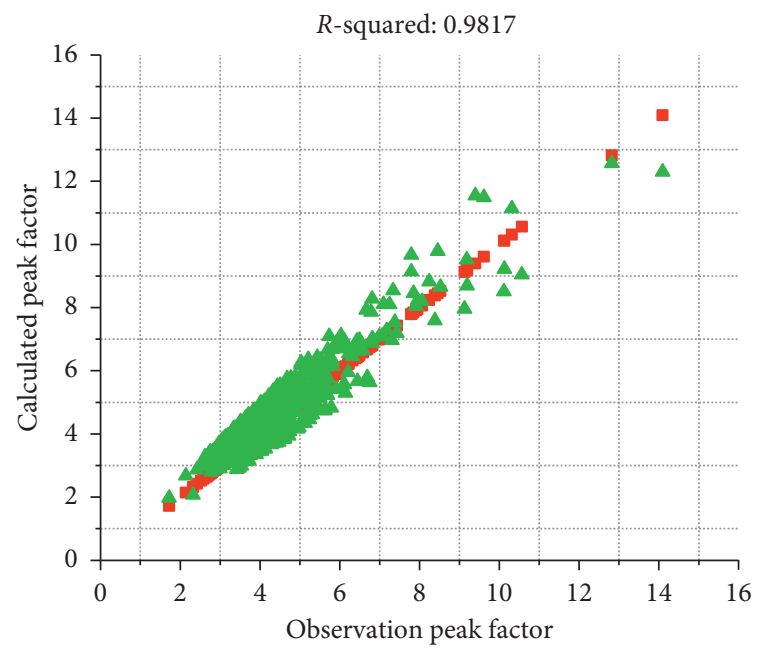

- Observation peak factor

- Stepwise search method

(a)

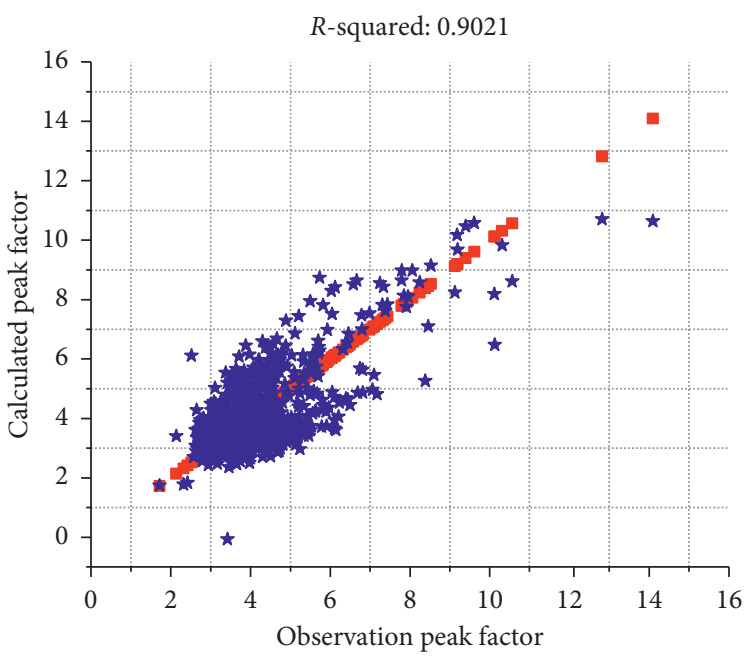

- Observation peak factor

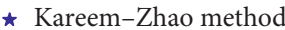

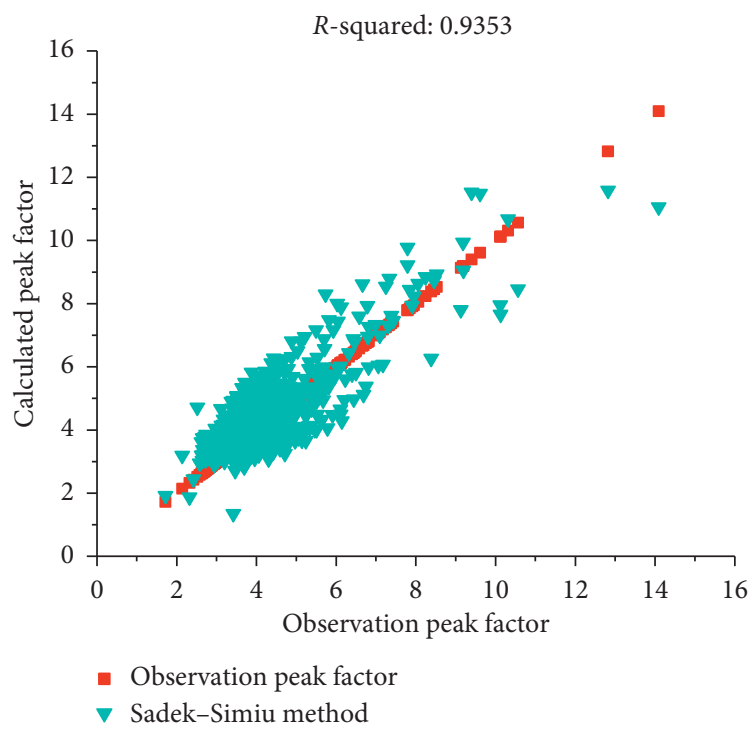

(c)

FIGURE 11: Peak factor of 808 measurement points calculated by different methods at a $90^{\circ}$ wind direction angle. (a) Comparison of stepwise search method peak factor and observed peak factor. (b) Comparison of Kareem-Zhao method peak factor and observed peak factor. (c) Comparison of Sadek-Simiu method peak factor and observed peak factor.

TABLE 3: Average error rates of the peak factor calculated by different methods under different working conditions.

\begin{tabular}{lccc}
\hline Working conditions & & Methods & \\
& Stepwise search method (\%) & Kareem-Zhao method (\%) & Sadek-Simiu method (\%) \\
\hline $90^{\circ}$ face D (left side face) & 9.72 & 16.94 & 13.92 \\
$90^{\circ}$ face A (windward) & 7.71 & 13.55 & 11.30 \\
$90^{\circ}$ face B (right side face) & 9.88 & 17.69 & 13.72 \\
$90^{\circ}$ face C (leeward) & 8.08 & 19.65 & 16.78 \\
$0^{\circ}$ & 5.15 & 17.16 & 10.76 \\
$22.5^{\circ}$ & 4.81 & 15.24 & 10.34 \\
$45^{\circ}$ & 10.87 & 17.24 & 13.69 \\
$90^{\circ}$ & 8.85 & 17.65 & 13.93 \\
$135^{\circ}$ & 7.23 & 16.24 & 11.76 \\
Overall average & $\mathbf{7 . 7 8}$ & $\mathbf{1 6 . 7 1}$ & $\mathbf{1 2 . 1 0}$ \\
\hline
\end{tabular}


TABLE 4: Peak factors with $99 \%$ guarantee rate calculated by the stepwise search method (on the $5^{\text {th }}$ measurement level at $90^{\circ}$ wind direction angle).

\begin{tabular}{|c|c|c|c|c|c|c|c|}
\hline \multicolumn{2}{|c|}{ Face D (left side face) } & \multicolumn{2}{|c|}{ Face A (windward) } & \multicolumn{2}{|c|}{ Face B (right side face) } & \multicolumn{2}{|c|}{ Face C (leeward) } \\
\hline Point number & Peak factor & Point number & Peak factor & Point number & Peak factor & Point number & Peak factor \\
\hline $5-1$ & 5.28 & $5-9$ & 3.38 & $5-17$ & 7.31 & $5-25$ & 5.20 \\
\hline $5-2$ & 4.96 & $5-10$ & 3.52 & $5-18$ & 5.75 & $5-26$ & 4.21 \\
\hline $5-3$ & 4.53 & $5-11$ & 3.52 & $5-19$ & 5.47 & $5-27$ & 4.73 \\
\hline $5-4$ & 5.09 & $5-12$ & 3.53 & $5-20$ & 4.20 & $5-28$ & 4.15 \\
\hline $5-5$ & 5.46 & $5-13$ & 3.46 & $5-21$ & 3.87 & $5-29$ & 4.12 \\
\hline $5-6$ & 4.61 & $5-14$ & 3.45 & $5-22$ & 4.92 & $5-30$ & 4.76 \\
\hline $5-7$ & 6.81 & $5-15$ & 3.45 & $5-23$ & 4.96 & $5-31$ & 4.99 \\
\hline $5-8$ & 5.96 & $5-16$ & 3.45 & $5-24$ & 5.48 & $5-32$ & 3.54 \\
\hline Average & 5.34 & Average & 3.47 & Average & 5.24 & Average & 4.46 \\
\hline
\end{tabular}

TABLE 5: Guarantee rates corresponding to the peak factor of 3.5 calculated by the stepwise search method (on the $5^{\text {th }}$ measurement level at a $90^{\circ}$ wind direction angle).

\begin{tabular}{|c|c|c|c|c|c|c|c|}
\hline \multicolumn{2}{|c|}{ Face D (left crosswind side) } & \multicolumn{2}{|c|}{ Face A (windward side) } & \multicolumn{2}{|c|}{ Face B (right crosswind side) } & \multicolumn{2}{|c|}{ Face C (leeward side) } \\
\hline Point number & Guarantee rate & Point number & Guarantee rate & Point number & Guarantee rate & Point number & Guarantee rate \\
\hline $5-1$ & 0.9899 & $5-9$ & 0.9998 & $5-17$ & 0.9831 & $5-25$ & 0.9966 \\
\hline $5-2$ & 0.9939 & $5-10$ & 0.9999 & $5-18$ & 0.9886 & $5-26$ & 0.9980 \\
\hline $5-3$ & 0.9926 & $5-11$ & 1.0000 & $5-19$ & 0.9959 & $5-27$ & 0.9985 \\
\hline $5-4$ & 0.9911 & $5-12$ & 1.0000 & $5-20$ & 0.9931 & $5-28$ & 0.9922 \\
\hline $5-5$ & 0.9896 & $5-13$ & 1.0000 & $5-21$ & 0.9935 & $5-29$ & 0.9967 \\
\hline $5-6$ & 0.9913 & $5-14$ & 0.9996 & $5-22$ & 0.9954 & $5-30$ & 0.9995 \\
\hline $5-7$ & 0.9876 & $5-15$ & 0.9980 & $5-23$ & 0.9945 & $5-31$ & 1.0000 \\
\hline $5-8$ & 0.9881 & 5-16 & 0.9966 & 5-24 & 0.9930 & $5-32$ & 0.9990 \\
\hline Average & 0.9905 & Average & 0.9992 & Average & 0.9921 & Average & 0.9976 \\
\hline
\end{tabular}

Gaussian pressure. Table 5 lists the guarantee rate corresponding to the peak factor of 3.5 of measurement points on the $5^{\text {th }}$ measurement level at $90^{\circ}$ wind direction angle. When the peak factor is 3.5 , the corresponding unilateral guarantee rate of the Gaussian model is $99.98 \%$, and the corresponding bilateral guarantee rate is $99.96 \%$ (see Table 1 ). The average guarantee rates of measurement points on the windward side, crosswind side, and leeward side are 99.92\%, 99.13\%, and $99.76 \%$, respectively, indicating that the same peak factor cannot ensure equivalent reliability for wind pressures with strong non-Gaussian properties. Usually, the absolute values of the extreme pressures increase when the nonGaussian character strengthens, and more sample points exceed the specified quantile value, thus leading to a smaller guarantee rate. For the target high-rise building investigated in this study, the non-Gaussian feature of wind pressures becomes increasingly obvious in the order of windward side $<$ leeward side $<$ crosswind side.

\section{Conclusions}

Based on reliability theory, a stepwise search method for solving the peak factor of non-Gaussian wind pressure is proposed in this study. The rescaled range analysis method, which quantitatively describes the nodal breakpoints of nonrandom walks of random signals in hydraulic engineering, is introduced to determine the optimal sectional capacity $n^{*}$. The gradual independent segmentation method is introduced to ensure independence between adjacent extremum values. To verify the feasibility of the proposed method and carry out comparisons with other peak factor estimation methods, the high-rise building (Shanghai World Financial Center) is taken as an example to obtain wind pressures on the claddings through wind tunnel tests. The main conclusions of this study are listed as follows:

(1) The accuracy of the peak factor calculated by the stepwise search method was confirmed to be better than that of the Sadek-Simiu method and KareemZhao method, and the peak factor estimated by the stepwise search method was comparable with that obtained by the Sadek-Simiu method. The estimated peak factor obtained by the stepwise search method is the closest to the observed value. The average error rates calculated by the stepwise search method are generally no more than $10 \%$, while the other methods achieve larger error rates within $20 \%$. The Kareem-Zhao method also overestimates the peak factor for most measurement points.

(2) For the particular section near the quadrilateral, the non-Gaussian feature of fluctuating wind pressures is prominent at the leading edge airflow separation area in the crosswind side, as well as both the leeward and the windward corner cuts. The non-Gaussian feature of wind pressures strengthens following the sequence of windward side $<$ leeward side $<$ crosswind side. In the case of $45^{\circ}$ wind direction angle, the 
two leeward surfaces exhibit strong non-Gaussian characteristic, even stronger than the two crosswind surfaces at $0^{\circ}$ wind direction angle.

(3) Generally, for a certain guarantee rate, the peak factor increases when the non-Gaussian feature strengthens and vice versa; however, special cases may exist.

\section{Data Availability}

The data (float type, can be saved as in .mat file or .txt file) of pressure tests on a rigid model of Shanghai World Financial Center, and the data generated during research used to support the findings of this study were supplied by State Key Laboratory of Disaster Reduction in Civil Engineering at Tongji University in China under license and can be obtained from the corresponding author upon request.

\section{Disclosure}

Any opinions and concluding remarks presented here are entirely those of the authors.

\section{Conflicts of Interest}

The authors declare that there are no conflicts of interest regarding the publication of this paper.

\section{Acknowledgments}

The work described in this study was supported by the Fostering Project of Innovation Team in Interdisciplinary Areas of Shanghai Science and Technology Commission (General Topic no. 03DZ12039). The wind tunnel test data of this study come from this project, which provides a guarantee for the completion of this study.

\section{References}

[1] P. Huang, M. Gu, and Z. C. Shi, "Discussion about the magnitude of the factor of peak value in wind tunnel test," Structural Engineers, vol. 4, pp. 16-19, 1997, in Chinese.

[2] Y. Quan, Bo. Yao, and M. Gu, "Research progress of wind reliability of high-rise buildings," Journal of Tongji University, vol. 43, no. 6, pp. 807-815, 2015.

[3] A. Kareem and J. Zhao, "Analysis of non-Gaussian surge response of tension leg platforms under wind loads," Journal of Offshore Mechanics and Arctic Engineering, vol. 116, no. 3, pp. 137-144, 1994.

[4] F. Sadek and E. Simiu, "Peak non-Gaussian wind effects for database-assisted low-rise building design," Journal of Engineering Mechanics, vol. 128, no. 5, pp. 530-539, 2002.

[5] M. F. Huang, W. Lou, C. M. Chan, N. Lin, and X. Pan, "Peak distributions and peak factors of wind-induced pressure processes on tall buildings," Journal of Engineering Mechanics, vol. 139, no. 12, pp. 1744-1756, 2013.

[6] A. G. Davenport, "Note on the distribution of the largest value of a random function with application to gust loading," Proceedings of the Institution of Civil Engineers, vol. 28, no. 2, pp. 187-196, 1964.
[7] D. K. Kwon and A. Kareem, "Peak factors for non-Gaussian load effects revisited," Journal of Structural Engineering, vol. 137, no. 12, pp. 1611-1619, 2011.

[8] M. Liu, X. Z. Chen, and Q. S. Yang, "Estimation of peak factor of non-Gaussian wind pressures by improved moment-based hermite model," Journal of Engineering Mechanics, vol. 143, no. 7, 2017.

[9] N. J. Cook, "Towards better estimation of extreme winds," Journal of Wind Engineering and Industrial Aerodynamics, vol. 9, pp. 3295-3323, 1982.

[10] Y. Quan, F. Wang, and M. Gu, "A method for estimation of extreme values of wind pressure on buildings based on the generalized extreme-value theory," Mathematical Problems in Engineering, vol. 2014, Article ID 926253, 22 pages, 2014.

[11] D. Wu, Study on wind effect uncertainty and wind-resistant design of large-span roofs, $\mathrm{PhD}$ Thesis, Harbin Institute of Technology, Harbin, China, 2012.

[12] M. W. Zhao and M. Gu, "Extreme wind pressure estimation based on the $r$ largest order statistics model," Chinese Journal of Theoretical and Applied Mechanics, vol. 42, no. 6, pp. 1074-1082, 2010, in Chinese.

[13] Y. Quan, M. Gu, B. Chen, and Y. Tamura, "Study on the extreme value estimating method of non-Gaussian wind pressure," Chinese Journal of Theoretical and Applied Mechanics, vol. 42, no. 3, pp. 560-566, 2010, in Chinese.

[14] B. Yao, Y. Quan, and M. Gu, "Combination method of wind load about high-rise buildings based on probability analysis," Journal of Tongji University, vol. 44, pp. 1032-1037, 2016, in Chinese.

[15] Y. Quan, H. H. Qiu, Z. W. Zhang, and M. Gu, "Wind tunnel test study on wind load of overhanging horizontal sunshade of high-rise buildings," Journal of Building Structures7 pages, 2020, in Chinese.

[16] Y. Luo, G. Q. Huang, Q. S. Yang, and Y. J. Tian, “Calculation of peak non-Gaussian wind pressures based on high-order moments," Journal of Building Structures, vol. 39, pp. 146-152, 2018, in Chinese.

[17] G. Huang and X. Chen, "Wind load effects and equivalent static wind loads of tall buildings based on synchronous pressure measurements," Engineering Structures, vol. 29, no. 10, pp. 2641-2653, 2007.

[18] X. Zhuang, X. Dong, J. M. Ding, and Y. M. Zhen, "NonGaussian features of wind pressure fluctuations on a rectangular high-rise building," Journal of Building Structures, vol. 37, no. suppl1, pp. 13-18, 2016, in Chinese.

[19] J. R. Wu and Q. X. Zheng, "Reliability analysis of wind-induced response of high-rise buildings with effect of joint distribution of wind speed and direction," Journal of Building Structures, vol. 38, no. 10, pp. 88-94, 2017, in Chinese.

[20] M. Gu and F. Ye, "Characteristics of wind pressure amplitude on super-tall buildings," Journal of Tongji University, vol. 34, no. 2, pp. 143-149, 2006, in Chinese.

[21] F. Ye and M. Gu, "Frequency characteristics of wind pressure on super-tall buildings," Journal of Tongji University, vol. 34, no. 3, pp. 285-290, 2006, in Chinese.

[22] Y. Tang, Research on the wind-excited vibrations and staticequivalent wind loads of torsionally coupled high-rise buildings, PhD Thesis, Tongji University, Shanghai, China, 2006.

[23] T. Ohkuma and A. Kanaya, "On the correlation between the shape of rectangular cylinders and characteristics of fluctuating lifts on them," Proceedings of $5^{\text {th }}$ Symposium on Wind Effects on Structures, pp. 147-154, Tokyo, Japan, 1978. 
[24] H. Choi, "Study on fluctuating wind force for 3-dimensional response analyses of tall buildings with rectangular crosssection," Thesis, Tokyo University, Tokya, Japan, 1990.

[25] R. L. Wardlaw and G. F. Moss, A Standard Tall Building Model for the Comparison of Simulated Natural Winds in Wind Tunnels, Commonwealth Advisory Aeronautical Research Council Report CC-662, Ottawa, Canada, 1970.

[26] W. H. Melbourne, "Comparison of measurements on the CAARC standard tall building model in simulated model wind flows," Journal of Wind Engineering and Industrial Aerodynamics, vol. 6, no. 1-2, pp. 73-88, 1980.

[27] N. J. Jamieson, P. Carpenter, and P. D. Cenek, "Wind induced external pressure on a tall building with various corner configurations," Journal of Wind Engineering and Industrial Aerodynamics, vol. 41-44, no. 1-3, pp. 2401-2412, 1992.

[28] J. Smagorinsky, "General circulation experiments with the primitive equations," Monthly Weather Review, vol. 91, no. 3, pp. 99-164, 1963.

[29] S. Huang, Q. S. Li, and S. Xu, "Numerical evaluation of wind effects on a tall steel building by CFD," Journal of Constructional Steel Research, vol. 63, no. 5, pp. 612-627, 2008.

[30] A. Elshaer, H. Aboshosha, G. Bitsuamlak, A. El Damatty, and A. Dagnew, "LES evaluation of wind-induced responses for an isolated and a surrounded tall building," Engineering Structures, vol. 115, pp. 179-195, 2016.

[31] P. Huang, M. Gu, and Y. Quan, "Wind tunnel test research on CAARC standard tall building model," Chinese Quarterly Mechanics, vol. 29, no. 4, pp. 627-633, 2008.

[32] H. Tanaka and N. Lawen, "Test on the CAARC standard tall building model with a length scale of 1:1000," Journal of Wind Engineering and Industrial Aerodynamics, vol. 25, no. 1, pp. 15-29, 1986.

[33] A. K. Dagnew and G. T. Bitsuamlak, "LES evaluation of wind pressures on a standard tall building with and without a neighboring building," in Proceedings of the Fifth International Symposium on Computational Wind Engineering, pp. 23-27, Chapel Hill, North Carolina, USA, May 2010.

[34] G. W. Alminhana, A. L. Braun, and A. M. Loredo-Souza, "A numerical-experimental investigation on the aerodynamic performance of CAARC building models with geometric modifications," Journal of Wind Engineering and Industrial Aerodynamics, vol. 180, pp. 34-48, 2018.

[35] F.-Q. Meng, B.-J. He, J. Zhu, D.-X. Zhao, A. Darko, and Z.-Q. Zhao, "Sensitivity analysis of wind pressure coefficients on CAARC standard tall buildings in CFD simulations," Journal of Building Engineering, vol. 16, pp. 146-158, 2018.

[36] M. S. Germi and H. E. Kalehsar, "Numerical investigation of interference effects on the critical wind velocity of tall buildings," Structures, vol. 30, pp. 239-252, 2021.

[37] D.-X. Zhao and B.-J. He, "Effects of architectural shapes on surface wind pressure distribution: case studies of oval-shaped tall buildings," Journal of Building Engineering, vol. 12, pp. 219-228, 2017.

[38] S. Chakraborty, S. K. Dalui, and A. K. Ahuja, "Wind load on irregular plan shaped tall building - a case study," Wind and Structures, vol. 19, no. 1, pp. 59-73, 2014.

[39] B. Bhattacharyya and S. K. Dalui, "Investigation of mean wind pressures on 'E' plan shaped tall building," Wind and Structures, vol. 26, pp. 99-114, 2018.

[40] M. Mallick, A. Mohanta, A. Kumar, and V. Raj, "Modelling of wind pressure coefficients on C-shaped building models," Modelling and Simulation in Engineering, vol. 2018, Article ID 6524945, 13 pages, 2018.
[41] Y. Kim and J. Kanda, "Characteristics of aerodynamic forces and pressures on square plan buildings with height variations," Journal of Wind Engineering and Industrial Aerodynamics, vol. 98, no. 8-9, pp. 449-465, 2010.

[42] K. Yuan, Y. Hui, and Z. Chen, "Effects of facade appurtenances on the local pressure of high-rise building," Journal of Wind Engineering and Industrial Aerodynamics, vol. 178, pp. 26-37, 2018.

[43] E. E. Peters, Fractal market analysis: Applying chaos theory to investment and economics, pp. 92-93, John Wiley \& Sons, Hoboken, NJ, USA, 1994.

[44] L. B. Xu and R. Lu, "R/S analysis to explore the nonlinearity of Chinese stock market," Prediction, vol. 18, no. 2, pp. 59-62, 1999.

[45] C. A. T. Ferro and J. Segers, "Inference for clusters of extreme values," Journal of the Royal Statistical Society: Series B (Statistical Methodology), vol. 65, no. 2, pp. 545-556, 2003.

[46] Y. Luo, Extreme value theory and its application on wind load and response of structure, $\mathrm{PhD}$ Thesis, Southwest Jiao tong University, Chengdu, China, 2018.

[47] D. Wu, Y. Wu, Q. S. Yang, and L. Chen, "Improved method of independent storms for extreme value estimation of nonGaussian wind pressure," Journal of Building Structures, vol. 35, pp. 151-156, 2014, in Chinese.

[48] Y. Sun, Y. Wu, Z. X. Lin, and Z. X. Lin, "Non-Gaussian features of fluctuating wind pressures on long-span roofs," Chinese Journal of Civil Engineering, vol. 29, pp. 9-15, 2007, in Chinese. 